\title{
On Soft Quantum B-Algebras and Fuzzy Soft Quantum B-Algebras
}

\author{
Xiongwei Zhang, ${ }^{1}$ Sultan Aljahdali, ${ }^{2}$ and Ahmed Mostafa Khalil $\mathbb{D}^{3}$ \\ ${ }^{1}$ School of Mathematics and Statistics, Yulin University, Yulin 719000, China \\ ${ }^{2}$ Department of Computer Science, College of Computers and Information Technology, Taif University, P. O. Box 11099, \\ Taif 21944, Saudi Arabia \\ ${ }^{3}$ Department of Mathematics, Faculty of Science, Al-Azhar University, Assiut 71524, Egypt
}

Correspondence should be addressed to Ahmed Mostafa Khalil; a.khalil@azhar.edu.eg

Received 20 August 2021; Accepted 15 September 2021; Published 16 October 2021

Academic Editor: Naeem Jan

Copyright (c) 2021 Xiongwei Zhang et al. This is an open access article distributed under the Creative Commons Attribution License, which permits unrestricted use, distribution, and reproduction in any medium, provided the original work is properly cited.

\begin{abstract}
This paper aims to make a combination between the quantum B-algebras (briefly, $\mathscr{X}$-Als) and two interesting theories (e.g., soft set theory and fuzzy soft set theory). Firstly, we propose the novel notions of soft quantum B-algebras (briefly, SQBB-As), a soft deductive system of $\mathbb{Q B}$-Ass, and deducible soft quantum B-algebras (briefly, $\mathbb{D} \mathbb{S} \mathbb{Q B}-A s$ ). Then, we discuss the relationship between $\mathbb{S Q B}$-As and $\mathbb{D S Q B}$-As. Furthermore, we investigate the union and intersection operations of $\mathbb{D} \mathbb{S Q B}$-As. Secondly, we introduce the notions of a fuzzy soft quantum B-algebras (briefly, $\mathbb{E S Q B}$-Ass), a fuzzy soft deductive system of $\mathbb{Q} B$-As, and present some characterizations of $\mathbb{E S Q B B}-\mathbb{A}$ s, along with several examples. Finally, we explain the basic properties of homomorphism image of $\mathbb{E S Q B}$-As.
\end{abstract}

\section{Introduction}

In 1999, Molodtsov [1] introduced the notion called soft sets (briefly, $\mathbb{S} S$ ) (i.e., which reduce the uncertainty and vagueness of knowledge). Maji et al. [2] presented the fuzzy soft sets (briefly, $\mathbb{E S S}$ ). Since then, many researchers studied further on $\mathbb{S} S$ and $\mathbb{E S S}$ as in the following published articles (e.g., [3-9]).

In 2014, Rump and Yang [10] proposed the notion of $\mathbb{Q} \mathbb{B}$-As (i.e., a partial ordered implication algebras). Rump $[11,12]$ investigated many implication algebras (for example, pseudo-BCK-algebras, po-groups, BL-algebras, MV-algebras, GPE-algebras, and resituated lattices). Botur and Paseka [13] studied filters on integral $\mathbb{Q} B-A s$, and Zhang et al. [14] established the quotient structures by using q-filters in $\mathbb{Q B B}$-As and investigated the relation between basic implication algebras and $\mathbb{Q B}$-As. Han et al. [15] constructed the unitality of $\mathbb{Q} \mathbb{B}$-As and explained the injective hulls of $\mathbb{Q B}$-As in [16]. By the framework of $\mathbb{Q} \mathbb{B}$-Ass, there are many published papers on $\mathbb{Q} B$-As (e.g., $[17-23])$.
Regarding these developments, as the motivation of this paper, we will combine $\mathbb{Q} \mathbb{B}$-As with $\mathbb{S} \mathbb{S}$ and $\mathbb{F} \mathbb{S} \mathbb{S}$ (i.e., enrich the previous work on hybrid soft set and fuzzy soft set theories algebras with quantum structures). We introduce the notions of $\mathbb{S Q B}$-As and the soft deductive system of $\mathbb{Q} B$-As and consider the relation between $\mathbb{S Q B}-A s$ and $\mathbb{D S} \mathbb{Q} B-A s$. Furthermore, some conditions are given to ensure the operations union and intersection holds of soft deductive of $\mathbb{Q B}$-Ass. Then, we investigate the homomorphism image of deductive $\mathbb{S Q B}-\mathbb{A}$ s. Lastly, we define $\mathbb{F} \mathbb{Q} \mathbb{B}-A$ s and fuzzy soft deductive system of $\mathbb{Q B}$-As and give an example to illustrate its derive properties.

In the following, we have arranged the sections as follows. In Section 2, we briefly recall many notions related to $\mathbb{Q} B$-As, $\mathbb{S} S$, and $\mathbb{E S S}$ as indicated in Definitions 1-7, which are used in the sequel. In Section 3, we propose the notions of $\mathbb{S Q B}-A s$, soft deductive system of $\mathbb{Q B}-A s$, and $\mathbb{D S} \mathbb{Q} B$-As. In Section 4, we present the notions of $\mathbb{E S Q B}$-As and a fuzzy soft deductive system of $\mathbb{Q} \mathbb{B}$-As and discuss the homomorphism image of $\mathbb{E S Q B}-\mathbb{A}$ s. The conclusions are explained in Section 5. 


\section{Preliminaries}

We give some basic notions of $\mathbb{Q B B}$-As, $\mathbb{S} \mathbb{S}$, and $\mathbb{E S} \mathbb{S}$ before defining $\mathbb{S Q B}$-As in Section 3.

Definition 1 (cf. [10]).

(1) $\mathbb{Q} B$-As is a partially ordered set $(\mathscr{X}, \leq)$ with two binary operations $\longrightarrow$ and $\rightsquigarrow$ which satisfy $(\forall x, y, z \in \mathscr{X})$ :

$$
\begin{aligned}
y \longrightarrow z & \leq(x \longrightarrow y) \longrightarrow(x \longrightarrow z), \\
y \rightsquigarrow z & \leq(x \mapsto y) \rightsquigarrow(x \rightsquigarrow z), \\
y & \leq z \longrightarrow x \longrightarrow y \leq x \longrightarrow z, \\
x & \leq y \longrightarrow z \Longleftrightarrow y \leq x \rightsquigarrow z .
\end{aligned}
$$

(2) $\mathbb{Q} \mathbb{B}-\mathbb{A}$ is a commutative (briefly, $\mathbb{C} \mathbb{Q} \mathbb{B}-\mathbb{A}$ ) if $x \longrightarrow y=x \rightsquigarrow y(\forall x, y \in \mathscr{X})$.

(3) A subset $\mathscr{Y}$ of a $\mathbb{Q} B-\mathbb{A} \mathscr{X}$ is a subalgebra if $x \longrightarrow y, x \leftrightarrow y \in \mathcal{Y}(\forall x, y \in \mathscr{X})$.

In what follows, denote by $\mathscr{X}$ a $\mathbb{Q} \mathbb{B}$ - $\mathbb{A}$ unless otherwise specified.

Definition 2 (cf. [10]). Let $\mathscr{X}_{1}$ and $\mathscr{X}_{2}$ be two $\mathbb{Q B}$-As. Then, $\psi: \mathscr{X}_{1} \longrightarrow \mathscr{X}_{2}$ is a morphism of $\mathbb{Q B}-\mathbb{A} s$ if it satisfies $\left(\forall x, y \in \mathscr{X}_{1}\right)$ :

$$
\begin{gathered}
\psi(x \longrightarrow y) \leq \psi(x) \longrightarrow \psi(y), \\
\psi(x \rightsquigarrow y) \leq \psi(x) \rightsquigarrow \psi(y) .
\end{gathered}
$$

We say morphism $\psi$ is exact if the inequalities become equations.

Definition 3 (cf. [1]). Assume that $\mathscr{X}$ be a set and $\mathscr{K}$ be a set of parameters. $\mathcal{S}_{\mathscr{K}}$ (called $\mathbb{S} S$ ) is a mapping given by $\mathcal{S}: \mathscr{K} \longrightarrow 2^{\mathscr{X}}$ (i.e., $2^{\mathscr{X}}$ is the power set of $\mathscr{X}$ ).

Definition 4 (cf. [3]). Assume that $\mathcal{S}_{\mathscr{K}_{1}}$ and $\mathcal{S}_{\mathscr{K}}$ are two $\mathbb{S} \mathbb{S}$ over $\mathscr{X} . \mathcal{S}_{\mathscr{K}_{1}}$ is a subset of $\mathcal{S}_{\mathscr{K}_{2}}\left(\right.$ denoted by $\mathcal{S}_{\mathscr{K}_{1}} \widetilde{\subset} \mathcal{S}_{\mathscr{K}_{2}}$ ) if

(1) $\mathscr{K}_{1} \subset \mathscr{K}_{2}$

(2) For every $k \in \mathscr{K}_{1}, \mathcal{S}_{\mathscr{K}_{1}}(k)$ and $\mathcal{S}_{\mathscr{K}_{2}}(k)$ are identical approximations

Definition 5 (cf. [3]). Assume that $\mathcal{S}_{\mathscr{K}_{1}}, \mathcal{S}_{\mathscr{K}_{2}}$, and $\mathcal{S}_{\mathscr{K}_{3}}$ are three $\mathbb{S} S$ over $\mathscr{X} . \mathcal{S}_{\mathscr{K}_{3}}$ is the intersection of $\mathcal{S}_{\mathscr{K}_{1}}$ and $\mathcal{S}_{\mathscr{K}_{2}}$ (denoted by $\mathcal{S}_{\mathscr{K}_{3}}=\mathcal{S}_{\mathscr{K}_{1}} \widetilde{\cap} \mathcal{S}_{\mathscr{K}_{2}}$ ) if

(1) $\mathscr{K}_{3}=\mathscr{K}_{1} \cap \mathscr{K}_{2}$

(2) $\forall k \in \mathscr{K}_{3}, \mathcal{S}_{\mathscr{K}_{3}}(k)=\mathcal{S}_{\mathscr{K}_{1}}(k)$ or $\mathcal{S}_{\mathscr{K}_{2}}(k)$ (as both are same sets)

Definition 6 (cf. [3]). Assume that $\mathcal{S}_{\mathscr{K}_{1}}, \mathcal{S}_{\mathscr{K}}$, and $\mathcal{S}_{\mathscr{K}_{3}}$ are three $\mathbb{S} S$ over $\mathscr{X} \cdot \mathcal{S}_{\mathscr{K}_{3}}$ is called the union of $\mathcal{S}_{\mathscr{K}_{1}}$ and $\mathcal{S}_{\mathscr{K}_{2}}$ (denoted by $\mathcal{S}_{\mathscr{K}_{3}}=\mathcal{S}_{\mathscr{K}_{1}} \widetilde{\cup} \mathcal{S}_{\mathscr{K}_{2}}$ ) if

(1) $\mathscr{K}_{3}=\mathscr{K}_{1} \cup \mathscr{K}_{2}$.
(2) $k \in \mathscr{K}_{3}$,

$$
\mathcal{S}_{\mathscr{K}_{3}}(k)= \begin{cases}\mathcal{S}_{\mathscr{K}_{1}}(k), & k \in \mathscr{K}_{1} \backslash \mathscr{K}_{2} \\ \mathcal{S}_{\mathscr{K}_{2}}(k), \quad k \in \mathscr{K}_{2} \backslash \mathscr{K}_{1}, \\ \mathcal{S}_{\mathscr{K}_{1}}(k) \cup \mathcal{S}_{\mathscr{K}_{2}}(k), \quad k \in \mathscr{K}_{1} \cap \mathscr{K}_{2}\end{cases}
$$

Definition 7 (cf. [2]). $\mathbb{E S S}$ (called $\mathbb{F S S}) \hat{\mathcal{S}}_{\mathscr{K}}$ is a mapping given by $\hat{\mathcal{S}}: \mathscr{K} \longrightarrow I^{X}$ (i.e., $I^{X}$ is the set of all fuzzy sets [24] of $\mathscr{X})$.

\section{3. $S \mathbb{Q B}-A$ s}

We define the $\mathbb{S Q B B}$-As and give several examples based on $\mathbb{S Q B}$-As. Also, we will study the union and intersection operations between two $\mathbb{S Q B}$-As as follows .

Definition 8. $\mathcal{S}_{\mathscr{K}}$ is a $\mathbb{S Q Q B}$-As over $\mathscr{X}$ if $\mathcal{S}_{\mathscr{K}}(x)(\forall x \in \mathscr{K})$ are subalgebras of $\mathscr{X}$ (i.e., in case $\mathscr{K}=\mathscr{X}$ ).

\section{Example 1}

(1) Suppose $\mathscr{X}$ (i.e., $\mathscr{X}=\left\{k_{1}, k_{2}, k_{3}, 1\right\}$ ) with the order $k_{2}, k_{3}<k_{1}<1$. Now, we show, by Table 1 , the binary operation $\longrightarrow$.

Clearly, $\mathscr{X}$ is a $\mathbb{C Q B}-\mathbb{A}$. We define $\mathcal{S}_{\mathscr{K}}(\forall x \in \mathscr{K})$ (i.e., $\mathscr{K}=\mathscr{X}$ ) by

$\mathcal{S}_{\mathscr{K}}(x)=\left\{y \in \mathscr{X} \mid(x \longrightarrow y) \longrightarrow y \in\left\{k_{1}, 1\right\}\right\}$.

From Table 1 , we can get on $\mathcal{S}_{\mathscr{K}}\left(k_{1}\right)=\mathscr{X}, \mathcal{S}_{\mathscr{K}}\left(k_{2}\right)=$ $\mathcal{S}_{\mathscr{K}},\left(k_{3}\right)=\left\{k_{1}, k_{3}, 1\right\}$, and $\mathcal{S}_{\mathscr{K}}(1)=\mathscr{X}$, and then, $\mathcal{S}_{\mathscr{K}}(x)(x \in \mathscr{K})$ are all subalgebras of $\mathscr{X}$. Consequently, $\mathcal{S}_{\mathscr{K}}$ is a $\mathbb{S Q B}$-As over $\mathscr{X}$.

(2) Suppose $\mathscr{X}$ (i.e., $\mathscr{X}=\left\{k_{1}, k_{2}, k_{3}, 1\right\}$ ) with the order $k_{1}<k_{2}<k_{3}<1$. Now, we show, by Table 2 , the binary operation $\longrightarrow$.

Clearly, $\mathscr{X}$ is a $\mathbb{C} \mathbb{Q} B-A$. We define $\mathcal{S}_{\mathscr{K}}(\forall x \in \mathscr{K})$ (i.e., $\mathscr{K}=\mathscr{X})$ by

$$
\mathcal{S}_{\mathscr{K}}(x)=\left\{y \in \mathscr{X} \mid x \mathscr{R} y \Longleftrightarrow x \longrightarrow(x \longrightarrow y) \in\left\{k_{3}, 1\right\}\right\} .
$$

From Table 2, we can get on (HTML translation failed), and then, $\mathcal{S}_{\mathscr{K}}(x)(x \in \mathscr{K})$ are all subalgebras of $\mathscr{X}$. Consequently, $\mathcal{S}_{\mathscr{K}}$ is a $\mathbb{S} \mathbb{Q} B$-As over $\mathscr{X}$.

We ensure the operations (i.e., union and intersection) are holding on $\mathbb{S} \mathbb{Q} B$ - $A$ s by the following suggested theorem.

Theorem 1. Assume that $\mathcal{S}_{\mathscr{K}_{1}}$ and $\mathcal{S}_{\mathscr{K}_{2}}$ are $\mathbb{S Q B}$-As over $\mathscr{X}$. Then,

(1) If $\mathscr{K}_{3}=\mathscr{K}_{1} \cap \mathscr{K}_{2}$, then $\mathcal{S}_{\mathscr{K}_{3}}=\mathcal{S}_{\mathscr{K}_{1}} \widetilde{\cap} \mathcal{S}_{\mathscr{K}_{2}}$ is called $a$ $\mathbb{S} \mathbb{Q} B-A$ over $\mathscr{X}$

(2) If $\mathscr{K}_{1} \cap \mathscr{K}_{2}=\varnothing$, then $\mathcal{S}_{\mathscr{K}_{1}} \widetilde{\cup} \mathcal{S}_{\mathscr{K}_{2}}$ is called a $\mathbb{S Q \mathbb { B }}$-A over $\mathscr{X}$ 
TABLE 1: The binary operation $\longrightarrow$.

\begin{tabular}{lcccc}
\hline$\longrightarrow$ & $k_{1}$ & $k_{2}$ & $k_{3}$ & 1 \\
\hline$k_{1}$ & 1 & $k_{1}$ & $k_{1}$ & 1 \\
$k_{2}$ & 1 & 1 & $k_{1}$ & 1 \\
$k_{3}$ & 1 & 1 & $k_{1}$ & 1 \\
1 & $k_{1}$ & $k_{2}$ & $k_{3}$ & 1 \\
\hline
\end{tabular}

TABLE 2: The binary operation $\longrightarrow$.

\begin{tabular}{lcccc}
$\longrightarrow \longrightarrow$ & $k_{1}$ & $k_{2}$ & $k_{3}$ & 1 \\
\hline$k_{1}$ & 1 & 1 & 1 & 1 \\
$k_{2}$ & $k_{1}$ & $k_{2}$ & 1 & 1 \\
$k_{3}$ & $k_{1}$ & $k_{1}$ & 1 & 1 \\
1 & $k_{1}$ & $k_{1}$ & $k_{3}$ & 1 \\
\hline
\end{tabular}

Proof

(1) If $\mathscr{K}_{3}=\mathscr{K}_{1} \cap \mathscr{K}_{2}$ and by Definition 5 , we obtain $\mathcal{S}_{\mathscr{K}_{3}}(x)=\mathcal{S}_{\mathscr{K}_{1}}(x)$ or $\mathcal{S}_{\mathscr{K}_{3}}(x)=\mathcal{S}_{\mathscr{K}_{2}}(x)$, for all $x \in \mathscr{K}_{3}$. Since $\mathcal{S}_{\mathscr{K}_{1}}$ and $\mathcal{S}_{\mathscr{K}_{2}}$ are $\mathbb{S Q \mathbb { Q }}$-As over $\mathscr{X}$, which implies that $\mathcal{S}_{\mathscr{K}_{3}}$ is a $\mathbb{S} \mathbb{Q} \mathbb{B}$-As over $\mathscr{X}$, that is, $\mathcal{S}_{\mathscr{K}_{3}}(x)=\mathcal{S}_{\mathscr{K}_{1}}(x)$ or $\mathcal{S}_{\mathscr{K}_{3}}(x)=\mathcal{S}_{\mathscr{K}_{2}}(x)$ are both subalgebras of $\mathscr{X}\left(\in \mathscr{K}_{3}\right)$, therefore, $\mathcal{S}_{\mathscr{K}_{3}}=\mathcal{S}_{\mathscr{K}_{1}} \widetilde{\sim} \mathcal{S}_{\mathscr{K}_{2}}$ is a $\mathbb{S Q B}$-A over $\mathscr{X}$.

(2) If $\mathscr{K}_{3}=\mathscr{K}_{1} \cup \mathscr{K}_{2}$ and by Definition 6, we obtain

$$
\mathcal{S}_{\mathscr{K}_{3}}(x)=\left\{\begin{array}{l}
\mathcal{S}_{\mathscr{K}_{1}}(x), \quad x \in \mathscr{K}_{1} \backslash \mathscr{K}_{2}, \\
\mathcal{S}_{\mathscr{K}_{2}}(x), \quad x \in \mathscr{K}_{2} \backslash \mathscr{K}_{1}, \\
\mathcal{S}_{\mathscr{K}_{1}}(x) \cup \mathcal{S}_{\mathscr{K}_{2}}(x), \quad x \in \mathscr{K}_{1} \cap \mathscr{K}_{2}
\end{array}\right.
$$

For $x \in \mathscr{K}_{1} \backslash \mathscr{K}_{2}$ and since $\mathcal{S}_{\mathscr{K}_{1}}$ is a $\mathbb{S Q B B}-\mathbb{A}$, then we have $\mathcal{S}_{\mathscr{K}_{3}}(x)=\mathcal{S}_{\mathscr{K}_{1}}(x)$ is a subalgebra of $\mathscr{X}$. Similarly, for $x \in \mathscr{K}_{2} \backslash \mathscr{K}_{1}$, then $\mathcal{S}_{\mathscr{K}_{3}}(x)=\mathcal{S}_{\mathscr{K}_{2}}(x)$ is a subalgebra of $\mathscr{X}$ due to $\mathcal{S}_{\mathscr{K}_{2}}$ is a $\mathbb{S Q Q B}$-A. Again, for $\mathscr{K}_{1} \cap \mathscr{K}_{2}=\varnothing$, so $x \in \mathscr{K}_{1} \cap \mathscr{K}_{2}$ or $x \in \mathscr{K}_{2} \cap \mathscr{K}_{1}$, for all $x \in \mathscr{K}_{3}$. Thus, $\mathcal{S}_{\mathscr{K}_{3}}=$ $\mathcal{S}_{\mathscr{K}_{1}} \widetilde{\cup} \mathcal{S}_{\mathscr{K}_{2}}$ is a $\mathbb{S Q B}$-A over $\mathscr{X}$.

Remark 1. If $\mathscr{K}_{1} \cap \mathscr{K}_{2} \neq \varnothing$, then Theorem 1 (2) does not hold by the following example.

Example 2. Suppose $\mathscr{X}$ (i.e., $\mathscr{X}=\left\{0, k_{1}, k_{2}, k_{3}, k_{4}, 1\right\}$ ). Now, we show, by Tables 3 and 4 , the binary operations $\longrightarrow$ and $\rightsquigarrow$, respectively.

Clearly, $\mathscr{X}$ is a $\mathbb{C} \mathbb{Q} B-A$. Then,

(i) We define $\mathcal{S}_{\mathscr{K}_{1}}\left(\forall x \in \mathscr{K}_{1}\right)$ (i.e., $\left.\mathscr{K}_{1}=\mathscr{X}\right)$ by

$$
\begin{aligned}
\mathcal{S}_{\mathscr{K}_{1}}(x)= & \{y \in \mathscr{X} \mid x \mathscr{R} y \Longleftrightarrow x \longrightarrow(x \longrightarrow y) x \\
& \left.\rightsquigarrow(x \mapsto y) \in\left\{k_{3}, k_{4}, 1\right\}\right\} .
\end{aligned}
$$

From Table 3 , we can get $\mathcal{S}_{\mathscr{K}_{1}}(0)=\mathscr{X}$ and $\mathcal{S}_{\mathscr{K}_{1}}$ $\left(k_{1}\right)=\mathcal{S}_{\mathscr{K}_{1}}\left(k_{2}\right)=\mathcal{S}_{\mathscr{K}_{1}}\left(k_{3}\right)=\mathcal{S}_{\mathscr{K}_{1}}\left(k_{4}\right)=\mathcal{S}_{\mathscr{K}_{1}}(1)=$ $\left\{k_{3}, k_{4}, 1\right\}$, and then, $\mathcal{S}_{\mathscr{K}_{1}}(x)\left(x \in \mathscr{K}_{1}\right)$ are all subalgebras of $\mathscr{X}$. Consequently, $\mathcal{S}_{\mathscr{K}_{1}}$ is a $\mathbb{S Q B}$-As over $\mathscr{X}$.
TABLE 3: The binary operation $\longrightarrow$.

\begin{tabular}{lcccccc}
\hline$\longrightarrow$ & 0 & $k_{1}$ & (HTML translation failed) & $k_{3}$ & $k_{4}$ & 1 \\
\hline 0 & 1 & 1 & 1 & 1 & 1 & 1 \\
$k_{1}$ & 0 & $k_{2}$ & 0 & $k_{4}$ & 1 & 1 \\
$k_{2}$ & 0 & 0 & $k_{2}$ & $k_{4}$ & $k_{4}$ & 1 \\
$k_{3}$ & 0 & 0 & 0 & 1 & 1 & 1 \\
$k_{4}$ & 0 & 0 & 0 & $k_{4}$ & 1 & 1 \\
1 & 0 & 0 & 0 & $k_{4}$ & $k_{4}$ & 1 \\
\hline
\end{tabular}

TABLE 4: The binary operation $\rightsquigarrow$.

\begin{tabular}{llccccc}
\hline$\rightsquigarrow$ & 0 & $k_{1}$ & $k_{2}$ & $k_{3}$ & $k_{4}$ & 1 \\
\hline 0 & 1 & 1 & 1 & 1 & 1 & 1 \\
$k_{1}$ & 0 & 0 & 0 & 1 & 1 & 1 \\
$k_{2}$ & 0 & $k_{1}$ & $k_{2}$ & $k_{3}$ & $k_{4}$ & 1 \\
$k_{3}$ & 0 & 0 & 0 & 1 & 1 & 1 \\
$k_{4}$ & 0 & 0 & 0 & 1 & 1 & 1 \\
1 & 0 & 0 & 0 & $k_{3}$ & $k_{4}$ & 1 \\
\hline
\end{tabular}

(ii) We define $\mathcal{S}_{\mathscr{K}_{2}}\left(\forall x \in \mathscr{K}_{2}\right)$ (i.e., $\left.\mathscr{K}_{2}=\left\{k_{2}\right\}\right)$ by

$$
\mathcal{S}_{\mathscr{K}_{2}}(x)=\left\{y \in \mathscr{K}_{2} \mid x \mathscr{R} y \Longleftrightarrow x \longrightarrow y=k_{2}, x \rightsquigarrow y=k_{2}\right\} .
$$

From Table 4 , we can get $\mathcal{S}_{\mathscr{K}_{2}}\left(k_{2}\right)=\left\{k_{2}\right\}$ is the subalgebra of $\mathscr{X}$. Consequently, $\mathcal{S}_{\mathscr{K}_{2}}$ is a $\mathbb{S Q B}$-As over $\mathscr{X}$.

From (i) and (ii) and $\mathscr{K}_{1} \cap \mathscr{K}_{2}=\left\{k_{2}\right\} \neq \varnothing$, then we have $\mathcal{S}_{\mathscr{K}_{3}}\left(k_{2}\right)=\mathcal{S}_{\mathscr{K}_{1}}\left(k_{2}\right) \cup \mathcal{S}_{\mathscr{K}_{2}}\left(k_{2}\right)=\left\{k_{3}, k_{4}, 1\right\} \cup\left\{k_{2}\right\}=$

$\left\{k_{2}, k_{3}, k_{4}, 1\right\}$ is not a subalgebra over $\mathscr{X}$. Thus, $\mathcal{S}_{\mathscr{K}_{3}}$ is not a SQB-A.

3.1. Soft Deductive Systems of $\mathbb{S} \mathbb{Q B}$-Ass. Based on Definition 8 , we will propose the notion of soft deductive systems of $\mathbb{S Q B}-$ As as indicated below.

Definition 9. Assume that $\mathscr{X}=(\mathscr{X}, \longrightarrow, \rightsquigarrow, \leq)$ be a $\mathbb{S Q B}-\mathbb{A}$. A nonempty subset $\mathscr{D} \subseteq \mathscr{X}$ is a deductive system of $\mathscr{X}$ if it satisfies

(1) $\forall x \in \mathscr{D}, x \longrightarrow x \in \mathscr{D}, x \rightsquigarrow x \in \mathscr{D}$

(2) $\forall x, y \in \mathscr{X}, x \in \mathscr{D}, x \longrightarrow y \in \mathscr{D} \Longrightarrow y \in \mathscr{D}$

Definition 10. Let $\mathscr{X}$ be a $\mathbb{S} \mathbb{Q} B-\mathbb{A}$ and $\mathscr{Y}$ a subalgebra of $\mathscr{X}$. A subset $\mathscr{D}$ of $\mathscr{X}$ is a deductive system of $\mathscr{X}$ related to $\mathscr{Y}$ (i.e., $\mathscr{Y}$-deductive system of $\mathscr{X}$ ), denoted by $\mathscr{D} \bowtie \mathscr{Y}$, and satisfies the following two conditions:

(1) $\forall x \in \mathscr{D}, x \longrightarrow x \in \mathscr{D}, x \rightsquigarrow x \in \mathscr{D}$

(2) $\forall y \in \mathscr{Y}, x \in \mathscr{D}, x \longrightarrow y \in \mathscr{D} \Longrightarrow y \in \mathscr{D}$

Remark 2. According to Definitions 9 and 10, we obtain that any deductive system of $\mathscr{X}$ is $\mathscr{Y}$-deductive system if $\mathscr{Y}$ is a subalgebra of $\mathscr{X}$. 
The converse of Remark 2 does not hold by Example 3 (i.e., $\mathcal{Y}$ is a subalgebra of $\mathscr{X}$ and $\mathscr{Y}$-deductive system is not a deductive system).

Example 3. Suppose $\mathscr{X}$ (i.e., $\mathscr{X}=\left\{0, k_{1}, k_{2}, k_{3}, 1\right\}$ ) with partial order $0<k_{1}<k_{3}<1$ and $0<k_{1}<k_{2}<1$. Now, we show, by Tables 5 and 6 , the binary operations $\longrightarrow$ and $\rightsquigarrow$, respectively.

Clearly, $\mathscr{X}$ is a $\mathbb{C Q B}-\mathbb{A}$. Consider a subalgebra $\mathscr{Y}=$ $\left\{k_{1}, 1\right\}$ and a subset $\mathscr{D}=\left\{k_{1}, k_{2}, 1\right\}$; we can see that $\mathscr{D} \bowtie \mathscr{Y}$. However, $\mathscr{D}$ is not a deductive system of $\mathscr{X}$ since $k_{3} \longrightarrow 1=$ $1 \in \mathscr{D}$ and $k_{3} \notin \mathscr{D}$.

Definition 11. Assume that $\mathcal{S}_{\mathscr{K}}$ is a $\mathbb{S} \mathbb{Q} \mathbb{B}-\mathbb{A}$ over $\mathscr{X} . \mathcal{S}_{\mathscr{D}}$ (i.e., $\mathbb{S} S$ ) over $\mathscr{X}$ is a soft deductive system of $\mathcal{S}_{\mathscr{K}}$, denoted by $\mathcal{S}_{\mathscr{D}} \widetilde{\triangleright \triangleleft} \mathcal{S}_{\mathscr{K}}$, and satisfies the following two conditions:

(1) $\mathscr{D} \subseteq \mathscr{K}$

(2) $\forall x \in \mathscr{D}, \mathcal{S}_{\mathscr{D}}(x) \triangleright \triangleleft \mathcal{S}_{\mathscr{K}}(x)$

Now, we will give an example to illustrate Definition 11 as follows.

Example 4. Suppose $\mathscr{X}$ (i.e., $\mathscr{X}=\left\{k_{1}, k_{2}, k_{3}, k_{4}, 1\right\}$ ) with partial order $k_{1}<k_{2}<k_{3}<k_{4}<1$. Now, we show, by Tables 7 and 8 , the binary operations $\longrightarrow$ and $\rightsquigarrow$, respectively.

Clearly, $\mathscr{X}$ is a $\mathbb{C} \mathbb{Q} B-A$. We define $\mathcal{S}_{\mathscr{K}}(\forall x \in \mathscr{K})$ (i.e., $\mathscr{K}=\mathscr{X})$ by

$$
\mathcal{S}_{\mathscr{K}}(x)=\{y \in \mathscr{X} \mid x \mathscr{R} y \Longleftrightarrow(x \longrightarrow y) \rightsquigarrow y=1\} .
$$

From Tables 7 and 8 , we can get on $\mathcal{S}_{\mathscr{K}}\left(k_{1}\right)=\mathcal{S}_{\mathscr{K}}\left(k_{2}\right)=$ $1, \quad \mathcal{S}_{\mathscr{K}}\left(k_{3}\right)=\left\{k_{2}, 1\right\}, \quad \mathcal{S}_{\mathscr{K}}\left(k_{4}\right)=\left\{k_{2}, k_{3}, 1\right\}$, and $\mathcal{S}_{\mathscr{K}}(1)=\mathscr{X}$, and then, $\mathcal{S}_{\mathscr{K}}(x)(x \in \mathscr{K})$ are all subalgebras of $\mathscr{X}$. Consequently, $\mathcal{S}_{\mathscr{K}}$ is a $\mathbb{S} \mathbb{Q} \mathbb{B}$-As over $\mathscr{X}$. by

Next, for a subset $\mathscr{D}=\left\{k_{2}, k_{4}\right\}$, we define $\mathcal{S}_{\mathscr{D}}(\forall x \in \mathscr{D})$

$$
\mathcal{S}_{\mathscr{D}}(x)=\{1\} \cup\{y \in \mathscr{X} \mid y \leq x\} .
$$

Then, we obtain $\mathcal{S}_{\mathscr{D}}\left(k_{2}\right)=\left\{k_{1}, k_{2}, 1\right\} \triangleright \triangleleft\{1\}=\mathcal{S}_{\mathscr{K}}\left(k_{2}\right)$ and $\mathcal{S}_{\mathscr{D}}\left(k_{4}\right)=\mathscr{X} \triangleright \triangleleft\left\{k_{2}, k_{3}, 1\right\}=\mathcal{S}_{\mathscr{K}}\left(k_{4}\right)$. Consequently, $\mathcal{S}_{\mathscr{D}}$ is a soft deductive system of $\mathcal{S}_{\mathscr{K}}$.

Theorem 2. Assume that $\mathcal{S}_{\mathscr{K}}$ is a $\mathbb{S Q \mathbb { B }}-\mathbb{A}$ over $\mathscr{X}$ and $\mathcal{S}_{\mathscr{D}_{1}}$ and $\mathcal{S}_{\mathscr{D}_{2}}$ are two $\mathbb{S} S$. Then,

(1) If $\quad \mathscr{D}_{1} \cap \mathscr{D}_{2} \neq \varnothing$, then $\mathcal{S}_{\mathscr{D}_{1}} \widetilde{\nabla \triangleleft} \mathcal{S}_{\mathscr{K}}, \mathcal{S}_{\mathscr{D}_{2}} \widetilde{\nabla \triangleleft}$ $\mathcal{S}_{\mathscr{K}} \Longrightarrow \mathcal{S}_{\mathscr{D}_{1}} \widetilde{\cap} \mathcal{S}_{\mathscr{D}_{2}} \widetilde{\nabla \triangleleft} \mathcal{S}_{\mathscr{K}}$

(2) If $\mathscr{D}_{1} \cap \mathscr{D}_{2}=\varnothing$, then $\mathcal{S}_{\mathscr{D}_{1}} \widetilde{\nabla \triangleleft} \mathcal{S}_{\mathscr{K}}, \mathcal{S}_{\mathscr{D}_{2}} \widetilde{\nabla \triangleleft} \mathcal{S}_{\mathscr{K}} \Longrightarrow$ $\mathcal{S}_{\mathscr{D}_{1}} \widetilde{\cup} \mathcal{S}_{\mathscr{D}_{2}} \widetilde{\nabla \triangleleft} \mathcal{S}_{\mathscr{K}}$

\section{Proof}

(1) Follow from Definition 5.

(2) If $\mathcal{S}_{\mathscr{D}_{1}} \widetilde{\nabla} \mathcal{S}_{\mathscr{K}}, \mathcal{S}_{\mathscr{D}_{2}} \widetilde{\nabla} \mathcal{S}_{\mathscr{K}}$, then, by Definition 6, we have $\mathscr{D}_{3}=\mathscr{D}_{1} \cap \mathscr{D}_{2}$ (i.e., $x \in \mathscr{D}_{3}$ ), $\mathcal{S}_{\mathscr{D}_{1}} \widetilde{\cup} \mathcal{S}_{\mathscr{D}_{2}}=\mathcal{S}_{\mathscr{D}_{3}}$, and
TABLE 5: The binary operation $\longrightarrow$.

\begin{tabular}{lccccc}
$\longrightarrow \longrightarrow$ & 0 & $k_{1}$ & $k_{2}$ & $k_{3}$ & 1 \\
\hline 0 & 1 & 1 & 1 & 1 & 1 \\
$k_{1}$ & 0 & 1 & $k_{2}$ & 1 & 1 \\
$k_{2}$ & $k_{1}$ & $k_{1}$ & 1 & 1 & 1 \\
$k_{3}$ & 0 & $k_{1}$ & $k_{2}$ & 1 & 1 \\
1 & 0 & $k_{1}$ & $k_{2}$ & $k_{3}$ & 1 \\
\hline
\end{tabular}

TABLE 6: The binary operation $\rightsquigarrow$.

\begin{tabular}{lccccc}
\hline$\rightsquigarrow$ & 0 & $k_{1}$ & $k_{2}$ & $k_{3}$ & 1 \\
\hline 0 & 1 & 1 & 1 & 1 & 1 \\
$k_{1}$ & $k_{2}$ & 1 & $k_{2}$ & 1 & 1 \\
$k_{2}$ & 0 & $k_{1}$ & 1 & 1 & 1 \\
$k_{3}$ & 0 & $k_{1}$ & $k_{2}$ & 1 & 1 \\
1 & 0 & $k_{1}$ & $k_{2}$ & $k_{3}$ & 1 \\
\hline
\end{tabular}

TABLE 7: The binary operation $\longrightarrow$.

\begin{tabular}{lccccc}
\hline$\longrightarrow$ & $k_{1}$ & $k_{2}$ & $k_{3}$ & $k_{4}$ & 1 \\
\hline$k_{1}$ & 1 & 1 & 1 & 1 & 1 \\
$k_{2}$ & $k_{3}$ & 1 & 1 & 1 & 1 \\
$k_{3}$ & $k_{2}$ & $k_{2}$ & 1 & 1 & 1 \\
$k_{4}$ & $k_{2}$ & $k_{2}$ & $k_{c}$ & 1 & 1 \\
1 & $k_{1}$ & $k_{2}$ & $k_{3}$ & $k_{4}$ & 1 \\
\hline
\end{tabular}

TABLE 8: The binary operation $m$.

\begin{tabular}{lccccc}
\hline$\rightsquigarrow$ & $k_{1}$ & $k_{2}$ & $k_{3}$ & $k_{4}$ & 1 \\
\hline$k_{1}$ & 1 & 1 & 1 & 1 & 1 \\
$k_{2}$ & $k_{4}$ & 1 & 1 & 1 & 1 \\
$k_{3}$ & $k_{2}$ & $k_{2}$ & 1 & 1 & 1 \\
$k_{4}$ & $k_{1}$ & $k_{2}$ & $k_{3}$ & 1 & 1 \\
1 & $k_{1}$ & $k_{2}$ & $k_{3}$ & $k_{4}$ & 1 \\
\hline
\end{tabular}

$$
\mathcal{S}_{\mathscr{D}_{3}}(x)= \begin{cases}\mathcal{S}_{\mathscr{D}_{1}}(x), & x \in \mathscr{D}_{1} \backslash \mathscr{D}_{2} \\ \mathcal{S}_{\mathscr{D}_{2}}(x), & x \in \mathscr{D}_{2} \backslash \mathscr{D}_{1}, \\ \mathcal{S}_{\mathscr{D}_{1}}(x) \cup \mathcal{S}_{\mathscr{D}_{2}}(x), & x \in \mathscr{D}_{1} \cap \mathscr{D}_{2}\end{cases}
$$

Since $\mathscr{D}_{1} \cap \mathscr{D}_{2}=\varnothing$, we obtain either $x \in \mathscr{D}_{1} \backslash \mathscr{D}_{2}$ or $x \in \mathscr{D}_{2} \backslash \mathscr{D}_{1}$. Then, we have the following:

Case 1: if $x \in \mathscr{D}_{1} \backslash \mathscr{D}_{2}$, since $\mathcal{S}_{\mathscr{D}_{1}} \widetilde{\nabla \triangleleft} \mathcal{S}_{\mathscr{K}}$, then $\mathcal{S}_{\mathscr{D}_{3}}(x)=\mathcal{S}_{\mathscr{D}_{1}}(x) \triangleright \triangleleft \mathcal{S}_{\mathscr{K}}(x)$

Case 2: if $x \in \mathscr{D}_{2} \backslash \mathscr{D}_{1}$ and $\mathcal{S}_{\mathscr{D}_{2}} \widetilde{\triangleright \triangleleft} \mathcal{S}_{\mathscr{K}}$, then $\mathcal{S}_{\mathscr{D}_{3}}(x)=\mathcal{S}_{\mathscr{D}_{2}}(x) \triangleright \triangleleft \mathcal{S}_{\mathscr{K}}(x)$

Consequently, for all $x \in \mathscr{D}_{3}$, we have $\mathcal{S}_{\mathscr{D}_{3}}(x) \triangleright \triangleleft \mathcal{S}_{\mathscr{K}}(x)$, which implies that $\mathcal{S}_{\mathscr{D}_{1}} \widetilde{\cup} \mathcal{S}_{\mathscr{D}_{2}}=\mathcal{S}_{\mathscr{D}_{3}} \widetilde{\nabla} \mathcal{S}_{\mathscr{K}}$.

Remark 3. If $\mathscr{K}_{1} \cap \mathscr{K}_{2} \neq \varnothing$, then Theorem 2 (2) does not hold by the following example.

Example 5. Suppose $\mathscr{X}$ (i.e., $\left.\mathscr{X}=\left\{0, k_{1}, k_{2}, k_{3}, k_{4}, 1\right\}\right)$. Now, we show, by Table 9 , the binary operations $\longrightarrow$. 
TABLE 9: The binary operation $\longrightarrow$.

\begin{tabular}{lcccccc}
\hline$\longrightarrow$ & 0 & $k_{1}$ & $k_{2}$ & $k_{3}$ & $k_{4}$ & 1 \\
\hline 0 & 1 & 1 & 1 & 1 & 1 & 1 \\
$k_{1}$ & $k_{3}$ & 1 & $k_{2}$ & $k_{3}$ & $k_{2}$ & 1 \\
$k_{2}$ & $k_{4}$ & $k_{1}$ & 1 & $k_{2}$ & $k_{1}$ & 1 \\
$k_{3}$ & $k_{1}$ & $k_{1}$ & 1 & 1 & $k_{1}$ & 1 \\
$k_{4}$ & $k_{2}$ & 1 & 1 & $k_{2}$ & 1 & 1 \\
1 & 0 & $k_{1}$ & $k_{2}$ & $k_{3}$ & $k_{4}$ & 1 \\
\hline
\end{tabular}

Clearly, $\mathscr{X}$ is a $\mathbb{C Q B}-\mathbb{A}$. Then,

(i) We define $\mathcal{S}_{\mathscr{K}}(\forall x \in \mathscr{K})$ (i.e., $\mathscr{K}=\mathscr{X}$ ) by $\mathcal{S}_{\mathscr{K}}(x)=\left\{y \in \mathscr{X} \mid x \mathscr{R} y \Longleftrightarrow(x \longrightarrow y) \longrightarrow y \in\left\{k_{1}, k_{2}, 1\right\}\right\}$.

From Table 9, we can get $\mathcal{S}_{\mathscr{K}}(0)=\left\{k_{1}, k_{2}, 1\right\}$, $\mathcal{S}_{\mathscr{K}}\left(k_{1}\right)=\mathscr{X}, \mathcal{S}_{\mathscr{K}}\left(k_{2}\right)=\mathcal{S}_{\mathscr{K}}\left(k_{3}\right)=\left\{k_{1}, 1\right\}$, and $\mathcal{S}_{\mathscr{K}}\left(k_{4}\right)=\left\{k_{1}, k_{2}, k_{3}, 1\right\}, \mathcal{S}_{\mathscr{K}}(1)=\mathscr{X}$, and then, $\mathcal{S}_{\mathscr{K}}(x)(\forall x \in \mathscr{K})$ are all subalgebras of $\mathscr{X}$. Consequently, $\mathcal{S}_{\mathscr{K}}$ is a $\mathbb{S Q B}$-As over $\mathscr{X}$.

(ii) We define $\mathcal{S}_{\mathscr{K}_{1}}\left(\forall x \in \mathscr{K}_{1}\right)$ (i.e., $\mathscr{K}_{1}=\left\{k_{1}, k_{2}, k_{3}\right\}$ ) by

$\mathcal{S}_{\mathscr{K}_{1}}(x)=\{y \in \mathscr{X} \mid x \mathscr{R} y \Longleftrightarrow x \longrightarrow y=1\}$.

Then, we can get $\mathcal{S}_{\mathscr{K}_{1}}\left(k_{1}\right)=\left\{k_{1}, 1\right\} \triangleright \triangleleft X=\mathcal{S}_{\mathscr{K}}\left(k_{1}\right)$, $\mathcal{S}_{\mathscr{K}_{1}}\left(k_{2}\right)=\left\{k_{2}, 1\right\} \triangleright \triangleleft\{a, 1\}=\mathcal{S}_{\mathscr{K}}\left(k_{2}\right), \quad$ and $\mathcal{S}_{\mathscr{K}_{1}}$ $\left(k_{3}\right)=\left\{k_{2}, k_{3}, 1\right\} \triangleright \triangleleft\left\{k_{1}, 1\right\}$. Therefore, $\mathcal{S}_{\mathscr{K}_{1}}$ is a soft deductive system over $\mathcal{S}_{\mathscr{K}}$.

(iii) We define $\mathcal{S}_{\mathscr{K}_{2}}\left(\forall x \in \mathscr{K}_{2}\right)$ (i.e., $\left.\mathscr{K}_{2}=\left\{k_{1}\right\}\right)$ by

$\mathcal{S}_{\mathscr{K}_{2}}(x)=\left\{y \in \mathscr{X} \mid x \mathscr{R} y \Longleftrightarrow y \longrightarrow x=k_{1}\right\}$.

Then, we can get $\mathcal{S}_{\mathscr{K}_{2}}\left(k_{1}\right)=\left\{k_{2}\right.$, $\left.k_{3}, 1\right\} \triangleright \triangleleft X=\mathcal{S}_{\mathscr{K}_{2}}\left(k_{1}\right)$. Therefore, $\mathcal{S}_{\mathscr{K}_{2}}$ is a soft deductive system over $\mathcal{S}_{\mathscr{K}}$.

From (i)-(iii), we have $\mathcal{S}_{\mathscr{K}_{3}}=\mathcal{S}_{\mathscr{K}_{1}} \widetilde{\cup} \mathcal{S}_{\mathscr{K}_{2}}$ which is not a soft deductive system of $\mathcal{S}_{\mathscr{K}}$, where $\mathcal{S}_{\mathscr{K}_{3}}\left(k_{1}\right)=\mathcal{S}_{\mathscr{K}_{1}}\left(k_{1}\right) \cup \mathcal{S}_{\mathscr{K}_{2}}\left(k_{1}\right)=\left\{k_{1}, k_{2}, k_{3}, 1\right\}$ is not a $\mathcal{S}_{\mathscr{K}}(a)$-deductive system because $k_{2} \longrightarrow k_{4}=k_{1} \in\left\{k_{1}, k_{2}, k_{3}, 1\right\}$ and $k_{4} \notin\left\{k_{1}, k_{2}, k_{3}, 1\right\}$.

3.2. $\mathbb{D S Q B}$-As. We will give the notion of $\mathbb{D} S \mathbb{Q} \mathbb{B}$-As and investigate homomorphism image of $\mathbb{D S} \mathbb{Q} \mathbb{B}$-As as indicated below.

Definition 12. Assume that $\mathcal{S}_{\mathscr{K}}$ is a $\mathbb{S} \mathbb{Q} B-\mathbb{A}$ over $\mathscr{X}$. If $\mathcal{S}_{\mathscr{K}}(x)(\forall x \in \mathscr{K})$ is a deductive system of $\mathscr{X}$, then $\mathcal{S}_{\mathscr{K}}$ is called a $\mathbb{D S} \mathbb{Q} B-A$ over $X$.

Example 6 (continued from Example 1 (2)). Clearly, $\mathcal{S}_{\mathscr{K}}$ is $\mathbb{D S Q B}$-A over $\mathscr{X}$.

\section{Definition 13}

(1) Suppose $\mathscr{X}$ be a $\mathbb{Q} \mathbb{B}-\mathbb{A}$ with the greatest element 1 (i.e., $\mathscr{X}$ just only a poset); for any $x \in \mathscr{X}$, the order of element $x$ is defined as

$$
\mathcal{O}(x)=\min \{p, q \in N \mid x \stackrel{p}{\longrightarrow} x=1, x \stackrel{q}{\rightarrow} x=1\},
$$

where $N$ is a natural number and $x \longrightarrow p x=(((x \longrightarrow x) \longrightarrow \cdots) \longrightarrow x), \quad x \rightarrow x=$ $(((x \mapsto x) \rightsquigarrow \cdots) \rightsquigarrow x)$.

(2) If $p, q \in N$ does not exist to satisfy the above condition (i), then $x(\forall x \in \mathscr{X})$ is called infinite order.

Remark 4. Assume that $\mathcal{S}_{\mathscr{K}}$ and $\mathcal{S}_{\mathscr{K}}$ be two $\mathbb{S} \mathbb{Q} B$-As over $\mathscr{X}$ such that $\mathscr{K}_{1} \subseteq \mathscr{K} \subseteq \mathscr{X}$. If $\mathcal{S}_{\mathscr{K}}$ is a $\mathbb{D} S \mathbb{Q} \mathbb{B}-A$ over $\mathscr{X}$, then $\mathcal{S}_{\mathscr{K}_{1}}$ is a $\mathbb{D S Q \mathbb { B }}-\mathbb{A}$.

The converse of Remark 4 does not hold by the following Example 7.

Example 7 (continued from Example 2). We define $\mathcal{S}_{\mathscr{K}}(\forall x \in \mathscr{K})$ (i.e., $\left.\mathscr{K}=\mathscr{X}\right)$ by

$$
\mathcal{S}_{\mathscr{K}}(x)=\{y \in \mathscr{X} \mid \mathcal{O}(x)=\mathscr{O}(y)\} .
$$

Then, we get on $\mathcal{S}_{\mathscr{K}}(0)=\mathcal{S}_{\mathscr{K}}\left(k_{3}\right)=$ $\mathcal{S}_{\mathscr{K}}\left(k_{4}\right)=\mathcal{S}_{\mathscr{K}}\left(k_{1}\right)=\left\{0, k_{3}, k_{4}, 1\right\}, \mathcal{S}_{\mathscr{K}}\left(k_{1}\right)=\left\{k_{1}\right\}, \quad$ and $\mathcal{S}_{\mathscr{K}}\left(k_{2}\right)=\left\{k_{2}\right\}$. However, $k_{3} \longrightarrow k_{1}=0 \in\left\{0, k_{3}, k_{4}, 1\right\}$ and $k_{1} \notin\left\{0, k_{3}, k_{4}, 1\right\}$ imply that $\mathcal{S}_{\mathscr{K}}$ is not $\mathbb{D S Q Q B}$-A. If we take $\mathscr{K}_{1}=\left\{k_{3}, k_{4}, 1\right\} \subseteq \mathscr{K} \quad$ and we define $\mathcal{S}_{\mathscr{K}_{1}}=\{y \in$ $\mathscr{X} \mid \mathcal{O}(x)=\mathscr{O}(y)\}\left(\forall x \in \mathscr{K}_{1}\right)$, then $\mathcal{S}_{\mathscr{K}_{1}}$ is $\mathbb{D} \mathbb{S Q B}-\mathbb{A}$.

Definition 14. Assume that $\mathcal{S}_{\mathscr{K}}$ is $\mathbb{S Q \mathbb { B }}-\mathbb{A}$ over $\mathscr{X}$ with the greatest element 1 . If $\mathcal{S}_{\mathscr{K}}(x)=\mathscr{X}(\forall x \in \mathscr{K})$, then $\mathcal{S}_{\mathscr{K}}$ is called whole $\mathbb{D} S \mathbb{Q} B-A$.

Example 8. Suppose $\mathscr{X}$ (i.e., $\mathscr{X}=\left\{0, k_{1}, k_{2}, 1\right\}$ ) with partial order $0<k_{1}<k_{2}<1$. Now, we show, by Tables 10 and 11 , the binary operations $\longrightarrow$ and $\rightsquigarrow$, respectively.

Clearly, $\mathscr{X}$ is a $\mathbb{C} \mathbb{Q} B-A$. We define $\mathcal{S}_{\mathscr{K}}(\forall x \in \mathscr{K})$ (i.e., $\mathscr{K}=\mathscr{X})$ by

$$
\mathcal{S}_{\mathscr{K}}(x)=\{y \in \mathscr{X} \mid \mathcal{O}(x)=\mathscr{O}(y)\} .
$$

From Tables 10 and 11, we can get on $\mathcal{S}_{\mathscr{K}}(x)=\mathscr{X}(\forall x \in \mathscr{K})$. Thus, $\mathcal{S}_{\mathscr{K}}$ is a whole $\mathbb{D} \mathbb{S} \mathbb{Q} \mathbb{B}$-A over $\mathscr{X}$

Now, we will study homomorphism image of $\mathbb{D S} \mathbb{Q} B$-As by the following two theorems.

Theorem 3. Assume that $\psi: \mathscr{X} \longrightarrow \mathscr{Y}$ be a surjective exact morphism of $\mathbb{Q} \mathbb{B}-\mathbb{A}$ and $\mathscr{X}$ is a $\mathbb{Q} \mathbb{B}-\mathbb{A}$ s. If $\mathcal{S}_{\mathscr{K}}$ is a $\mathbb{D S} \mathbb{Q}$-A over $\mathscr{X}$, then $\psi\left(\mathcal{S}_{\mathscr{K}}\right)$ is also $\mathbb{D} \mathbb{Q} \mathbb{B}-\mathbb{A}$ over $\mathcal{Y}$.

Proof. Since $\mathcal{S}_{\mathscr{K}}(x)(x \in \mathscr{K})$ is a deductive system of $\mathscr{X}$ and $\psi$ is surjective, then $\psi\left(\mathcal{S}_{\mathscr{K}}\right)(x)=\psi\left(\mathcal{S}_{\mathscr{K}}(x)\right)$ is a deductive system of $\mathcal{Y}$ which implies that $\psi\left(\mathcal{S}_{\mathscr{K}}\right)$ is a $\mathbb{D} \mathbb{S} \mathbb{Q} B$-A over $\mathscr{X}$.

Theorem 4. Assume that $\psi: X \longrightarrow Y$ be a surjective exact morphism of $\mathbb{Q B}-\mathbb{A}$ and $\mathcal{S}_{\mathscr{K}}$ a $\mathbb{D S} \mathbb{Q} B-A$ over $X$. Then, 
TABLE 10: The binary operation $\longrightarrow$.

\begin{tabular}{lcccc}
\hline$\longrightarrow$ & 0 & $k_{1}$ & $k_{2}$ & 1 \\
\hline 0 & 1 & 1 & 1 & 1 \\
$k_{1}$ & $k_{1}$ & 1 & 1 & 1 \\
$k_{2}$ & $k_{1}$ & $k_{1}$ & 1 & 1 \\
1 & 0 & $k_{1}$ & $k_{2}$ & 1 \\
\hline
\end{tabular}

TABLE 11: The binary operation $m$.

\begin{tabular}{lcccc}
\hline$\rightsquigarrow$ & 0 & $k_{1}$ & $k_{2}$ & 1 \\
\hline 0 & 1 & 1 & 1 & 1 \\
$k_{1}$ & $k_{2}$ & 1 & 1 & 1 \\
$k_{2}$ & 0 & $k_{1}$ & 1 & 1 \\
1 & 0 & $k_{1}$ & $k_{2}$ & 1 \\
\hline
\end{tabular}

(1) If $\mathcal{S}_{\mathscr{K}}(x)=k e r(\psi)$, for all $x \in \mathscr{K}$, then $\psi\left(\mathcal{S}_{\mathscr{K}}\right)$ is the whole $\mathbb{D S Q B}-A$ over $Y$

(2) If is whole $\mathbb{D S Q B}-\mathbb{A}$ over $\mathscr{X}$, then $\psi\left(\mathcal{\delta}_{\mathscr{K}}\right)$ is the whole $\mathbb{D S Q B}$-A over Y

Proof

(1) Assume that $\delta_{\mathscr{K}}(x)=\operatorname{ker}(\psi)$, where $\operatorname{ker}(\psi)=\{x \in \mathscr{X} \mid \psi(x)=x \longrightarrow x, \quad \psi(x)=x \leftrightarrow x\}$. Since $\psi$ is surjective, then, from Theorem 3 , we have $\psi\left(\mathcal{S}_{\mathscr{K}}\right)(x)=\psi\left(\mathcal{S}_{\mathscr{K}}(x)\right)=\psi(\mathscr{X}) \quad=\mathscr{Y}(x \in \mathscr{K})$. Thus, $\psi\left(\mathcal{S}_{\mathscr{K}}\right)$ is the whole $\mathbb{D S Q B B}-\mathbb{A}$ over $\mathscr{Y}$.

(2) Clearly, $\delta_{\mathscr{K}}(x)=\mathscr{X}$ since $\mathcal{S}_{\mathscr{K}}$ is whole $\mathbb{D S Q B B}-\mathrm{A}$ over $\mathscr{X}(x \in \mathscr{K})$. Thus, $\psi\left(\mathcal{S}_{\mathscr{K}}\right)(x)=\psi\left(\mathcal{S}_{\mathscr{K}}(x)\right)=\psi(\mathscr{X})=\mathscr{Y}(x \in \mathscr{K})$. By Theorem 3, we have $\psi\left(\delta_{\mathscr{K}}\right)$ is the whole DSQB-A over $y$

\section{FSQBB-As}

We give the definition of $\mathbb{F S Q B}$-As; a concrete example is given to illustrate its derive properties. Furthermore, we study the homomorphism image and preimage of $\mathbb{F S Q B}$-As. Now, we first propose the definition of fuzzy quantum $\mathrm{B}$-algebra (briefly, $\mathbb{F} \mathbb{Q B}-\mathbb{A}$ ) as indicated below.

Definition 15. We call $\mathbb{F} \mathbb{Q B}-\mathbb{A}$ (or a fuzzy set $\widehat{\mu}$ in $\mathbb{Q} \mathbb{B}-\mathbb{A}$ ) if it satisfies $(\forall x, y \in \mathscr{X}, \mathscr{X}$ is $\mathbb{Q} \mathbb{B}-\mathbb{A})$ :

$$
\begin{aligned}
\widehat{\mu}(x \longrightarrow y) & \geq \min \{\widehat{\mu}(x), \widehat{\mu}(y)\}, \\
\widehat{\mu}(x \mapsto y) & \geq \min \{\widehat{\mu}(x), \widehat{\mu}(y)\} .
\end{aligned}
$$

Definition 16 . We call $\widehat{\mu}$ is a fuzzy deductive system of $\mathscr{X}$ if it satisfies $(\forall x, y \in \mathscr{X})$ :

$$
\begin{aligned}
\widehat{\mu}(x \longrightarrow x) & \geq \widehat{\mu}(x), \\
\widehat{\mu}(x \mapsto x) & \geq \widehat{\mu}(x), \\
\widehat{\mu}(y) & \geq \min \{\widehat{\mu}(x \longrightarrow y), \widehat{\mu}(x)\} .
\end{aligned}
$$

Definition 17. Assume that $\hat{S}_{\mathscr{K}}$ be a $\mathbb{F S S}$ over $\mathscr{X}$. Then,

(1) If there exists $\hat{\mu} \in \mathscr{K}$ such that $\hat{\mathcal{S}}_{\mathscr{K}}[\mu]$ is a $\mathbb{F} \mathbb{Q} \mathbb{B}-\mathbb{A}$ (i.e., fuzzy deductive system) in a $\mathbb{Q} \mathbb{B}$ - $\mathbb{A}$ over $\mathscr{X}$, then $\hat{\mathcal{S}}_{\mathscr{K}}$ is called a $\hat{\mathcal{S}}_{\mathscr{K}}$-A (i.e., fuzzy soft deductive system $\mathbb{E S D S}$ ) which depends on a parameter set $\widehat{\mu}$ over $\mathscr{X}$

(2) If $\hat{\delta}_{\mathscr{K}}[\mu]$ is a $\mathbb{F Q \mathbb { B }}$ - $\mathbb{A}$ (i.e., fuzzy deductive system) of $\mathscr{X}$ based on all parameters, then we say that $\hat{\delta}_{\mathscr{K}}$ is a $\mathbb{F S Q B - A}$ (i.e., $\mathbb{F S D S}$ ) of $X$

In the following, a concrete example is given to illustrate Definition 17.

Example 9. Suppose that there are five-class cars:

$$
X=\{\text { BMW, Audi, Toyota, Jeep, Cadilac }\} .
$$

Let $\oplus$ and $\otimes$ be two soft machines to characterize two cars, defined by the following manner.

BMW $\oplus x=$ Cadilac, forall $x \in \mathscr{X}$,

$$
\text { Audi } \oplus y= \begin{cases}\text { Jeep, } & y=\text { BMW, } \\ \text { Cadilac, }, & y \in\{\text { Audi, Toyouta, Jeep, Cadilac }\},\end{cases}
$$

Toyota $\oplus z= \begin{cases}\text { Toyota, } & z=\text { BMW, } \\ \text { Jeep, } & z=\text { Audi, } \\ \text { Cadilac, } & z \in\{\text { Toyota, Jeep, Cadilac }\},\end{cases}$

$$
\text { Jeep } \oplus s=\left\{\begin{array}{l}
\text { Toyota, } s=\text { BMW, } \\
\text { Jeep, } s \in\{\text { Audi, Toyoya }\}, \\
\text { Cadilac, } s \in\{\text { Jeep, Cadilac }\},
\end{array}\right.
$$

Cadilac $\oplus t=\left\{\begin{array}{l}\text { BMW, } t=\text { BMW, } \\ \text { Audi, } t=\text { Audi, } \\ \text { Toyota, } t=\text { Toyota, } \\ \text { Jeep, } t=\text { Jeep, } \\ \text { Cadilac, } t=\text { Cadilac, }\end{array}\right.$

BMW $\otimes x=$ Cadilac forall $x \in \mathscr{X}$,

Audi $\otimes y=\left\{\begin{array}{l}\text { Jeep, } y=\text { BMW, } \\ \text { Cadilac, } y \in\{\text { Audi, Toyouta, Jeep, Cadilac }\},\end{array}\right.$

Toyota $\otimes z=\left\{\begin{array}{l}\text { Jeep, } z \in\{\text { BMW, Audi }\}, \\ \text { Cadilac, } z \in\{\text { Toyouta, Jeep, Cadilac }\},\end{array}\right.$

Jeep $\otimes s=\left\{\begin{array}{l}\text { Audi, } s=\text { BMW, } \\ \text { Jeep, } s \in\{\text { Audi, Toyouta }\}, \\ \text { Cadilac, } s \in\{\text { Jeep, Cadilac }\},\end{array}\right.$

Cadilac $\otimes t=\left\{\begin{array}{l}\text { BMW, } t=\text { BMW, } \\ \text { Audi, } t=\text { Audi, } \\ \text { Toyota, } t=\text { Toyota, } \\ \text { Jeep, } t=\text { Jeep, } \\ \text { Cadilac, } t=\text { Cadilac. }\end{array}\right.$ 
Then, $(\mathscr{X}, \oplus, \otimes, \leq)$ is a $\mathbb{Q B}-\mathbb{A}$. Now, we consider a set of parameters: $\widehat{\mu}=($ Excellent, Good, Moderate $) \in \in \mathscr{K}$. Then, we have the following:

(1) We define $\hat{\mathcal{S}}_{\mathscr{K}}[\widehat{\mu}]$ over $\mathscr{X}$ (i.e., $\hat{\delta}_{\mathscr{K}}$ [Excellent], $\hat{\mathcal{S}}_{\mathscr{K}}\left[\right.$ Good], and $\hat{\mathcal{S}}_{\mathscr{K}}$ [Moderate] are fuzzy sets) by Table 12 .

Therefore, we can see that $\hat{\delta}_{\mathscr{K}}$ [Excellent], $\hat{\delta}_{\mathscr{K}}\left[\right.$ Good], and $\hat{\delta}_{\mathscr{K}}$ [Moderate] are all $\mathbb{E S Q B B}$-As based on parameters "Excellent," "Good," and "Moderate" over $\mathscr{X}$. Thus, $\hat{\mathcal{S}}_{\mathscr{K}}$ is a $\mathbb{E S Q B}$ - $\mathbb{A}$ over $\mathscr{X}$.

(2) We define $\hat{\mathcal{S}}_{\mathscr{K}}[\widehat{\mu}]$ over $\mathscr{X}$ (i.e., $\hat{\mathcal{S}}_{\mathscr{K}_{1}}$ [Excellent], $\hat{\mathcal{S}}_{\mathscr{K}_{1}}$ [Good], and $\hat{\mathcal{S}}_{\mathscr{K}_{1}}$ [Moderate] are fuzzy sets) by Table 13.

However, $\hat{\delta}_{\mathscr{K}}[\widehat{\mu}]$ is not a $\mathbb{E S Q B}-A$ based on a parameter "Excellent" over (HTML translation failed), where

$\hat{\mathcal{S}}_{\mathscr{K}_{1}}$ [Excellent] (Toyota $\left.\oplus \mathrm{BMW}\right)=\hat{\mathcal{S}}_{\mathscr{K}_{1}}$ [Excellent] (Toyota) $=0.1 ¥ 0.2=\min \{0.2,0.4\}=\min \left\{\widehat{\mathcal{S}}_{\mathscr{K}_{1}}\right.$ [Excellent] (Audi), $\hat{\delta}_{\mathscr{K}_{1}}$ [Excellent] (BMW)\}. Also, we obtain that $\hat{\delta}_{\mathscr{K}_{1}}[\widehat{\mu}]$ is a $\mathbb{E S Q B}$ - $\mathrm{A}$ based on both the parameter "Good" and "Moderate" over $\mathscr{X}$.

(3) We define $\hat{\delta}_{\mathscr{K}_{2}}[\hat{\mu}]$ over $\mathscr{X}$ (i.e., $\hat{\mathcal{S}}_{\mathscr{K}_{2}}$ [Excellent] and $\hat{\mathcal{S}}_{\mathscr{K}_{2}}$ [Good] are fuzzy sets) by Table 14 .

Then, $\hat{\mathcal{S}}_{\mathscr{K}_{2}}[\widehat{\mu}]$ is a $\mathbb{E S D S}$ on parameters "Excellent." However, $\hat{\delta}_{\mathscr{K}_{2}}[\mu]$ is not a fuzzy deductive system of $\mathscr{X}$ based on parameter "Good," where $\hat{\delta}_{\mathscr{K}_{2}}\left[\right.$ Good] $($ Toyota $)=0.3<0.5=\quad \min \left\{\hat{\mathcal{S}}_{\mathscr{K}_{2}}\right.$ [Good] (Jeep $\oplus$ Toyota), $\widehat{\mathcal{S}}_{\mathscr{K}_{2}}$ [Good] (Jeep)\}.

(4) We define $\hat{\delta}_{\mathscr{K}_{3}}[\widehat{\mu}]$ over $\mathscr{X}$ (i.e., $\hat{\mathcal{S}}_{\mathscr{K}_{3}}$ [Excellent] and $\widehat{\delta}_{\mathscr{K}_{2}}$ [Moderate] are fuzzy sets) by Table 15 .

Then, $\hat{\mathcal{S}}_{\mathscr{K}_{3}}[\widehat{\mu}]$ is a $\mathbb{E S D S}$ of $\mathscr{X}$.

Now, we will present several characterizations of ESQRB-As.

By Definition 17 , if $\hat{\mathcal{S}}_{\mathscr{K}}$ is a $\mathbb{E S Q B}$ - $\mathbb{A}$ of $\mathbb{Q B B}$ - $\mathbb{A}$ over $\mathscr{X}$ based on all parameters, then we say that $\hat{\delta}_{\mathscr{K}}$ is a $\mathbb{F S Q B}$-A of $\mathscr{X}$, that is,

$$
\begin{aligned}
\hat{\mathcal{S}}_{\mathscr{K}}[\widehat{\mu}](x \longrightarrow y) & \geq \min \left\{\hat{\mathcal{S}}_{\mathscr{K}}[\widehat{\mu}](x), \hat{\mathcal{S}}_{\mathscr{K}}[\widehat{\mu}](y)\right\}, \\
\hat{\mathcal{S}}_{\mathscr{K}}[\widehat{\mu}](x \rightsquigarrow y) & \geq \min \left\{\hat{\mathcal{S}}_{\mathscr{K}}[\widehat{\mu}](x), \hat{\mathcal{S}}_{\mathscr{K}}[\widehat{\mu}](y)\right\} .
\end{aligned}
$$

Proposition 1. Assume $\mathscr{X}$ be a $\mathbb{Q B}$-A. If $\hat{\mathcal{S}}_{\mathscr{K}}$ is $\mathbb{E S Q R B}-\mathbb{A}$ over $\mathscr{X}$, then, for all $t \in[0,1],\left(\hat{\mathcal{S}}_{\mathscr{K}}\right)_{t} \neq \varnothing$ is the subalgebra of $\mathscr{X}$, in which

$$
\left(\hat{\mathcal{S}}_{\mathscr{K}}\right)_{t}=\left\{\left(\hat{\mathcal{S}}_{\mathscr{K}}[\widehat{\mu}]\right)_{t} \mid \widehat{\mu} \in \mathscr{K}\right\} .
$$

Proof. Let $\left(\widehat{\mathcal{S}}_{\mathscr{K}}[\widehat{\mu}]\right)_{t} \neq \varnothing$. Then, $\forall x, y \in\left(\widehat{\mathcal{S}}_{\mathscr{K}}[\widehat{\mu}]\right)_{t}$; since $\hat{\mathcal{S}}_{\mathscr{K}}$ is a $\mathbb{E S Q R B}-A$, then $\hat{\mathcal{S}}_{\mathscr{K}}[\widehat{\mu}](x) \geq t, \hat{\mathcal{S}}_{\mathscr{K}}[\widehat{\mu}](y) \geq t$. So,
TABLe 12: Fuzzy sets $\hat{\delta}_{\mathscr{K}}[\widehat{\mu}]$ over $\mathscr{X}$.

\begin{tabular}{lccccc}
\hline$\hat{\mathcal{S}}_{\mathscr{K}}$ & BMW & Audi & Toyota & Jeep & Cadilac \\
\hline Excellent & 0.2 & 0.2 & 0.5 & 0.6 & 0.8 \\
Good & 0.1 & 0.2 & 0.3 & 0.5 & 0.7 \\
Moderate & 0.1 & 0.1 & 0.4 & 0.4 & 0.6 \\
\hline
\end{tabular}

TABLe 13: Fuzzy sets $\hat{\mathcal{S}}_{\mathscr{K}_{1}}[\hat{\mu}]$ over $\mathscr{X}$.

\begin{tabular}{lccccc}
\hline$\hat{\mathcal{S}}_{\mathscr{K}_{1}}$ & BMW & Audi & Toyota & Jeep & Cadilac \\
\hline Excellent & 0.4 & 0.2 & 0.1 & 0.6 & 0.8 \\
Good & 0.2 & 0.2 & 0.3 & 0.5 & 0.7 \\
Moderate & 0.1 & 0.1 & 0.4 & 0.5 & 0.9 \\
\hline
\end{tabular}

TABLE 14: Fuzzy sets $\hat{\delta}_{\mathscr{K}_{2}}[\hat{\mu}]$ over $\mathscr{X}$.

\begin{tabular}{lccccc}
\hline$\hat{\mathcal{S}}_{\mathscr{K}_{2}}$ & BMW & Audi & Toyota & Jeep & Cadilac \\
\hline Excellent & 0.2 & 0.2 & 0.2 & 0.2 & 0.6 \\
Good & 0.2 & 0.2 & 0.3 & 0.5 & 0.7 \\
\hline
\end{tabular}

TABLE 15: Fuzzy sets $\hat{\delta}_{\mathscr{K}_{3}}[\widehat{\mu}]$.

\begin{tabular}{lccccc}
\hline$\hat{\mathcal{S}}_{\mathscr{K}_{3}}$ & BMW & Audi & Toyota & Jeep & Cadilac \\
\hline Excellent & 0.3 & 0.3 & 0.3 & 0.3 & 0.3 \\
Moderate & 0.1 & 0.1 & 0.1 & 0.1 & 0.7 \\
\hline
\end{tabular}

$$
\begin{aligned}
\hat{\mathcal{S}}_{\mathscr{K}}[\hat{\mu}](x \longrightarrow y) & \geq \min \left\{\hat{\mathcal{S}}_{\mathscr{K}}[\widehat{\mu}](x \longrightarrow y), \hat{\mathcal{S}}_{\mathscr{K}}[\widehat{\mu}](x \rightsquigarrow y)\right\} \\
& \geq \min \left\{\hat{\mathcal{S}}_{\mathscr{K}}[\hat{\mu}](x), \hat{\mathcal{S}}_{\mathscr{K}}[\hat{\mu}](y)\right\} \geq t .
\end{aligned}
$$

Similarly, we have $\hat{\delta}_{\mathscr{K}}[\widehat{\mu}](x \rightsquigarrow y) \geq t$. Therefore, $x \longrightarrow y, x \rightsquigarrow y \in\left(\hat{\mathcal{S}}_{\mathscr{K}}[\widehat{\mu}]\right)_{t}$. This implies that $\left(\hat{\mathcal{S}}_{\mathscr{K}}[\widehat{\mu}]\right)_{t}$ is the subalgebra of $\mathscr{X}$.

Analogously, we can get Proposition 2 as follows.

Proposition 2. Assume that $\mathcal{S}_{\mathscr{K}_{1}}$ and $\mathcal{S}_{\mathscr{K}_{2}}$ are two $\mathbb{E S Q B}-\mathbb{A}$ over $\mathscr{X}$. Then, $\mathcal{S}_{\mathscr{K}_{1}} \widetilde{\cap} \mathcal{S}_{\mathscr{K}_{2}}$ and $\mathcal{S}_{\mathscr{K}_{1}} \widetilde{\cup} \mathcal{S}_{\mathscr{K}_{2}}$ are $\mathbb{E S Q B}$-As over $x$.

Definition 18. Let $(\alpha, \beta)$ be a fuzzy soft map from $\mathbb{Q} \mathbb{B}-\mathbb{A}$ over $\mathscr{X}$ to $\mathbb{Q B}$-A over $\mathcal{Y}$. Then,

(1) If $\alpha$ is an exact morphism from $\mathscr{X}$ to $\mathscr{Y}$, then $(\alpha, \beta)$ is called a $\mathbb{E S Q B}$-A exact morphism from $\mathscr{X}$ to $\mathcal{Y}$

(2) If $\alpha$ is an isomorphism from $\mathscr{X}$ to $\mathscr{Y}$ and $\beta$ is a bijective from $\mathscr{K}_{1}$ to $\mathscr{K}_{2}$, then $(\alpha, \beta)$ is a called an isomorphism between $\mathbb{E S Q B B}$-As

Proposition 3. Let $X$ and $\mathcal{Y}$ be two $\mathbb{Q B}$-As. $\mathcal{S}_{\mathscr{K}}$ is a $\mathbb{F S Q B - A}$ over $Y$ and $(\alpha, \beta)$ a $\mathbb{E S Q B}$-A exact morphism from $X$ to $\mathcal{Y}$; then, $(\alpha, \beta)^{-1} \mathcal{S}_{\mathscr{K}}$ is $\mathbb{E S Q B B}-\mathbb{A}$ over $X$. 
Proof. For $\widehat{\mu} \in \beta^{-1}(\mathscr{K})$,

$$
\begin{aligned}
\alpha^{-1} & \left(\mathcal{S}_{\mathscr{K}}\right)[\widehat{\mu}](x \longrightarrow y) \\
& =\mathcal{S}_{\mathscr{K}} \beta[\widehat{\mu}](\alpha(x \longrightarrow y)) \\
& =\mathcal{S}_{\mathscr{K}} \beta[\widehat{\mu}](\alpha(x) \longrightarrow \alpha(y)) \\
& \geq \min \left\{\mathcal{S}_{\mathscr{K}} \beta[\widehat{\mu}] \alpha(x), \mathcal{S}_{\mathscr{K}} \beta[\widehat{\mu}] \alpha(y)\right\} \\
& =\min \left\{\alpha^{-1}\left(\mathcal{S}_{\mathscr{K}}\right)[\widehat{\mu}](x), \alpha^{-1}\left(\mathcal{S}_{\mathscr{K}}\right)[\widehat{\mu}](x)\right\} .
\end{aligned}
$$

Consequently, $(\alpha, \beta)^{-1} \mathcal{S}_{\mathscr{K}}$ is a $\mathbb{F} \mathbb{Q Q B}-\mathbb{A}$ over $\mathscr{X}$. Similarly, we can get Proposition 4 as follows.

Proposition 4. Let $\mathscr{X}$ and $\mathcal{Y}$ be two $\mathbb{Q B}-\mathbb{A} s . \mathcal{S}_{\mathscr{K}}$ is a $\mathbb{F S Q \mathbb { B }}-A$ s over $\mathcal{X}$ and $(\alpha, \beta)$ a $\mathbb{E S Q Q B}-A$ s isomorphism from $\mathscr{X}$ to $\mathcal{Y}$; then, $(\alpha, \beta) \mathcal{S}_{\mathscr{K}}$ is the $\mathbb{E} \mathbb{S} \mathbb{Q B}-A$ s over $\mathcal{Y}$.

\section{Conclusions}

In this paper, we introduce the concept of $\mathbb{S} \mathbb{Q} \mathbb{B}$-As, and some examples are given to illustrate this definition. Also, we investigate the union and intersection operations between two $\mathbb{S Q B}-A$ s and give some conditions for the operation holds. With the help of the definition of $\mathbb{S Q B}-A$ s, we define soft deductive systems of $\mathbb{S Q B}$-As and then investigate the relation between them. As a further step, we define $\mathbb{D S Q \mathbb { B }}$-As and investigate the homomorphism image of $\mathbb{D S Q R B}$-As. Moreover, we define $\mathbb{E S Q B}$-As. Finally, a concrete example is given to illustrate its derive properties; besides, homomor-

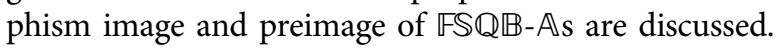

As a future work, it makes sense to apply $\mathbb{S} \mathbb{Q B}$-As to medical diagnosis (for example, $[25,26]$ ) in practice. Furthermore, it would be interesting if we study hybrid soft lattice-ordered quantum B-algebras.

\section{Data Availability}

No data were used to support this study.

\section{Conflicts of Interest}

The authors declare that they have no conflicts of interest.

\section{Acknowledgments}

Sultan Aljahdali acknowledges Taif University Researchers Supporting Project (no. TURSP2020/73), Taif University, Taif, Saudi Arabia. This work was supported by the science and technology project of Yulin City (CXY-2020-007).

\section{References}

[1] D. Molodtsov, "Soft set theory-first results," Computers \& Mathematics with Applications, vol. 37, no. 4-5, pp. 19-31, 1999.

[2] P. K. Maji, R. Biswas, and A. R. Roy, "Fuzzy soft sets," Journal of Fuzzy Mathematics, vol. 9, no. 3, pp. 589-602, 2001.

[3] P. K. Maji, R. Biswas, and A. R. Roy, "Soft set theory," Computers \& Mathematics with Applications, vol. 45, no. 4-5, pp. 555-562, 2003.

[4] N. Cagman and S. Enginoglu, "Soft set theory and unit decision making," European Journal of Operational Research, vol. 207, no. 2, pp. 848-855, 2010.
[5] A. M. Khalil, S.-G. Li, H.-X. Li, and S.-Q. Ma, "Possibility $\mathrm{m}$-polar fuzzy soft sets and its application in decision-making problems," Journal of Intelligent and Fuzzy Systems, vol. 37, no. 1, pp. 929-940, 2019.

[6] A. M. Khalil and N. Hassan, "Inverse fuzzy soft set and its application in decision making," International Journal of Information and Decision Sciences, vol. 11, no. 1, pp. 73-92, 2019.

[7] A. M. Khalil, S.-G. Li, H. Garg, H. Li, and S. Ma, "New operations on interval-valued picture fuzzy set, interval-valued picture fuzzy soft set and their applications," IEEE Access, vol. 7, pp. 51236-51253, 2019.

[8] H. Lu, A. M. Khalil, W. El-Gayar, and M. A. El-Gayare, "A new type of generalized picture fuzzy soft set and its application in decision making," Journal of Intelligent and Fuzzy Systems, vol. 40, no. 6, pp. 12459-12475, 2021.

[9] C. Cheng, Z. Cao, and F. Xiao, "A generalized belief intervalvalued soft set with applications in decision making," Soft Computing, vol. 77, no. 24, pp. 9339-9350, 2020.

[10] W. Rump and Y. C. Yang, "Non-commutative logical algebras and algebraic quantales," Annals of Pure and Applied Logic, vol. 165, no. 2, pp. 759-785, 2014.

[11] W. Rump, “Quantum B-algebras," Central European Journal of Mathematics, vol. 11, pp. 1881-1899, 2013.

[12] W. Rump, "Quantum B-algebras: their omnipresence in algebraic logic and beyond," Soft Computing, vol. 21, no. 10, pp. 2521-2529, 2017.

[13] M. Botur and J. Paseka, "Filters on some classes of quantum B-algebras," International Journal of Theoretical Physics, vol. 54, no. 12, pp. 4397-4409, 2015.

[14] X. Zhang, R. Borzooei, and Y. Jun, "Q-Filters of quantum B-algebras and basic implication algebras," Symmetry, vol. 10, no. 11 , p. $573,2018$.

[15] S. Han, X. Xu, and F. Qin, "The unitality of quantum B-algebras," International Journal of Theoretical Physics, vol. 57, no. 5, pp. 1582-1590, 2018.

[16] S. Han, R. Wang, and X. Xu, "On the injective hulls of quantum B-algebras," Fuzzy Sets and Systems, vol. 369, no. 15, pp. 114-121, 2019.

[17] W. Rump and Y. Yang, "Hereditary arithmetics," Journal of Algebra, vol. 468, pp. 214-252, 2016.

[18] F. Pan, "Dual quantum B-algebras," Soft Computing, vol. 23, no. 16, pp. 6813-6817, 2019.

[19] L. C. Ciungu, "Monadic classes of quantum B-algebras," Soft Compting, vol. 25, pp. 1-14, 2021.

[20] C. Xia, "On the finite embeddability property for quantum B-algebras," Mathematica Slovaca, vol. 69, no. 4, pp. 721-728, 2019.

[21] Q. Zhan, "Some operators on quantum B-algebras," Symmetry, vol. 13, no. 8, p. 1381, 2021.

[22] S. Han and X. Xu, "A few notes on quantum B-algebras," Studia Logica, 2021.

[23] S. Han and Z. Bao, "Locally unital quantum B-algebras," Journal of Algebra and Its Applications, 2021.

[24] L. A. Zadeh, "Fuzzy sets," Information and Control, vol. 8, no. 3, pp. 338-353, 1965.

[25] A. M. Khalil, S. G. Li, Y. Lin, H. X. Li, and S. G. Ma, “A new expert system in prediction of lung cancer disease based on fuzzy soft sets," Soft Computing, vol. 24, pp. 14179-14207, 2020.

[26] N. Hassan, O. R. Sayed, A. M. Khalil, and M. A. Ghany, "Fuzzy soft expert system in prediction of coronary artery disease," International Journal of Fuzzy Systems, vol. 19, no. 5, pp. 1546-1559, 2017. 